\section{G.I. Mihalas, \\ D. Lungeanu}

partment of Medical Informatics, University of Medicine and Pharmacy, inisoara, Rumania.

\title{
Education and Training
}

\section{Strategy for Medical Informatics Education at the University of Medicine and Pharmacy in Timisoara}

\begin{abstract}
Our strategy for adapting a curriculum in medical informatics for medical students is presented. After a brief overview, some specific conditions are addressed, which account for some peculiarities of the objectives of our courses and of the design of the curriculum, including its integration in the overall medical curriculum. Some efforts concerning examinations and some specific educational tools are then discussed. The paper also deals with particular features concerning medical informatics education for dentistry and pharmacy students as well as for postgraduate courses, paying special attention to the doctoral level. Finally, future perspectives of medical informatics education are discussed.

The paper does not present a list of successes and achievements. We had, and still have several difficulties (not al ways related to financial drawbacks), which required a particuilar approach; the word "strategy" in the title is also meant to cover these aspects.
\end{abstract}

Keywords: Medical Informatics, Medical Education, Curriculum, Dental Informatics, Informatics in Pharmacy.

came aware of our role in medical informatics education in Rumania.

This paper aims to present our strategy to adapt a curriculum elaborated in a developed country to our specific conditions and to integrate this discipline into the overalleducation of medical students.

\section{Specific Conditions}

The section on "Objectives" is not the first one approached, but our specific conditions yielded some changes even at that level. Some of the main considerations are:

- our students have a good background in basic sciences: while the communist regimealmost broke down the industrial sector, education was much less affected. Our medical students have to pass a difficultentrance examination comprising physics, chemistry and biology; thus, we can often rely on their background and use formal approaches, formulae, various graphs, problems, etc.;

- the educational system is not modular: the whole study period is divided into six "years of study", each comprising two semesters of 15 weeks of courses followed by an examination period of 3-4 weeks. Students are obliged to attend most of the disciplines (very few are optional), and have to pass all the examinations to successfully complete that year of study; try (most of them founded in the same period) shared our experience we be- 
- poor background in computers: few high schools were able to offer computer facilities to their pupils, so most of our students have their first computer experience in our laboratory; thus, we had to dedicate some hours to the basics of computers, including PC main components, operating systems and computer networks - as courses, accompanied by laboratory work on using utility programs, main Windows operations and use of a text editor. Unfortunately, we spend $20 \%$ of our course time and $25 \%$ of laboratory time on these background topics, which should (ideally) be covered at the high school level.

- large number of students: Rumania used to have only six (now 10) schools of medicine (for over 22 millioninhabitants), enrolling around 2500 Rumanian medical students per year, about 250 in dentistry and 200 in pharmacy. There are also foreign students, so we estimate the average number of students per year of study (in Timisoara) to be over 450 in medicine, over 100 in dentistry and around 50 in pharmacy; this yields over 600 students per year. They are divided into groups of about12 students, and have to share our 10 computers of a LAN between 24 students. This will change next year when a new LAN will be purchased for our department and the desired ratio 1:1 may be achieved. This "crowd" imposed a reasonable list of tasks to be performed during laboratory work and also increased the responsibility of the assistant-professors who supervise the laboratories.

- time limitations: when first accepted as a discipline within the medical curriculum, medical informatics had 15 hours for courses and 15 hours for laboratory work. Nowadays, there are 30 hours of each, but even with this extent we eventually cover our objectives (since the basic knowledge of computers and biostatistics represents over $40 \%$ percent of this time). However, we integrated biostatistics within the laboratory work. Several other medical schools have also included biostatistics into their medical informatics curricula (see, for instance, a whole chapter in [2]).

- no background in medicine: our course takes place in the very first semester of study and there is no othermodule onmedical informatics during the total of 12 semesters over six years of undergraduate medical studies. This placing of medical informatics at the beginning of medical studies (which is not specific for our country) entails some shortcomings: e.g. presenting ECG processing before studying the physiology of the heart, or discussion of decision support in diagnosis before having basic medical knowledge; etc. An integration such as done in London at CHIME [3] seems ideal but, for us, a second module would cover most of these shortcomings.

- poor level of IT use in medical units: the process of introducing computers in hospitals was extremely slow, mainly due to financial reasons. The clinical departments which succeeded in acquiring one or two computers mainly belong to university hospitals; the applications are various: databases, statistical analysis, medical documentation, signal or image processing, etc. But there are few places with a structured departmental information system, and we cannot speak yet of a hospital information system, or of a primary care information system. Since concordance between the contents of a lecture, the tasks to be performed during laboratory work, and the real needs for clinical practice represent a "must" for teaching and a strong motivation for learning, we feel unsatisfied with the chapter on infor. mation systems. However, the healthcare system in Rumania is currently the subject of substantial reform and a large project has just started. Our present students will be among those who will need more details about information systems.

\section{Objectives and Principles}

We established a set of objectives to be reached by our students, which proved to be close to the thorough list of objectives made by the members of the EDUCTRA Concerted Action [4]. So here we only briefly comment on the priorities and some principles to be followed:

- the present students will start practiv ing medicine after six years, which for computer technology is a long timeduring which improvements at expected; thus, it is better to empha size the principlesunderlyingmedical applications so thatstudents will more easily understand and assimilatenew developments;

- computers can not replace physi cians or nurses, and students should always be aware of the limitations which are specific for any application;

- use of computers has become a necessity and contributes to improved organization and thinking;

- useful results can be obtained only when based on good quality of data; students should be aware of the importance of data quality, integrity, protection and security;

- standards will play an increasingly important role in medical activities and students should understand this role;

- computer networks will dramatically change several medical activities, especially documentation and communication. 


\section{Designing the Curriculum}

Whereas establishing the objectives and principles has a rather theoretical character, when designing the curriculum the most appropriate ways must be found to reach the objectives and to present details about the contents of each part of a lecture or laboratory work.

As pedagogical principles require, teaching should start "from simple to complex", so that sequencing topics becomes important [5]. For interdisciplinary fields there are several ways to divide the topics into chapters, i.e., we have a multiaxial classification system. Moreover, each chapter can be scaled from simple to complex. The best way to achieve deep understanding would be to cover the contents twice: first, during the first or second year ("preclinical"), comprising basic knowledge and applications, with a clear view of the main streams of medical applications of computers; and the second time, during clinical studies when the student would better understand each application. But, due to time limitations and because we do not have a second module, we had to adopt a curriculum which starts with basic knowledge about computers (structure, operating systems, networking), followed by: (medical) databases (types of fields, codification, files etc.), biostatistics (parameters, distribution, estimation, tests, correlation and regression, risk analysis), biosignals (acquisition, methods of processing, ECG, EEG), image processing (sampling and quantization, types of processing, medical imaging - tomography, etc.), decision support (logical methods, Bayes rule, pattern recognition, basics of logic, expert systems, classification analysis), computers in medical documentation, education and research (includingmodeling and simulation; simple deterministic models, stochastic models, modeling structures, non-linear models, neural networks).

\section{Integration}

We were aware from the beginning that this feature is fundamental for a new discipline to become a permanent part of the medical educational process. Effort was made for all possible links to other disciplines since our staff is made up of mathematicians, engineers and physicists. Collaboration with our physician colleagues from preclinical and clinical departments succeeded partially, and the experience was mutually useful. We introduced several examples from basic disciplines such as: biostatistics of nonlinear correlation illustrated with biophysical phenomena, regulatory systems and biosignals used on physiology, simulation of biochemical enzymatic reactions, methods for analysing anatomical structures, stochastic approaches in genetics, models in immunology, parameter estimation in pharmacology, etc. Integration with clinical disciplines was not more difficult but it was too early for our students. They were able to understand the structure of a patient record, types of fields, importance of unique identification, codification and nomenclatures, etc., but most of the clinical examples were accepted as true and students usually have no comments or queries, even when obvious discrepancies within data occurred.

We were not able (yet) to associate an appropriate laboratory work for our course on information systems due to the lack of a corresponding real system (but a "virtual praxis" is now under construction). The same is true for "pattern recognition", which isincluded in our course on medical decisionmaking and presented along with statistical methods for decision support.

We expect to achieve a better integration next year when a new "multimedia laboratory" will be in use, and our contacts with other clinical and preclinical disciplines will have increased.

\section{Testing and Examination}

From a cybernetic viewpoint, the educational process can be regarded as a regulated system which has feedback represented by all types of examinations, in order to adapt the teaching, so that the students' knowledge is as close as possible to the desired objectives. All pedagogical books dedicate a chapter to this topic (docimology). We would not say that this feature was completely neglected, but we found few papers on this topic $[6,7]$. However, we created a large set of "problems and questions" for examinations because we consider that a problem-based examination better represents students' comprehension and ability to use their knowledge than a multiple-choice questionnaire.

We should note here that Rumania was isolated for a long time, with little information on events in the rest of the world. Now that we have - in principle - access to all information there is a trend to copy systems, but little care is taken to adapt them to particular conditions; perhaps even worse - to copy only what is convenient with no consideration for integration. The importance of a discipline within the global framework of medical education has often been estimated by the proportion of questions from that discipline included in general examinations, e.g. board licence examinations. In the materials available to us, medical informatics was poorly represented.

Our examinations normally comprise a practical and a theoretical examination. For the practical students perform some basic computer operations, create a data file and use a statistical package for data processing, and interpret the results. The theoretical part comprises a set of problems concentrated on biostatistics and risk analysis, biosignals and images, medical decision making, (biochemical) model building and fractals. There is also a set of questions/topics with 
short and simple answers. Finally, the students also address 2-3 theoretical topics which require a more detailed presentation.

Multiple-choice questionnaires were once used but the present type of examination, requiring students to explicate their knowledge is better suited to our purposes.

\section{Specific Educational Tools}

From the beginning we considered a good demonstration of the changes a computer can bring might be done using computers wherever possible. Our efforts here concentrated on computers for lectures, and on designing specific computer programs for laboratory work.

\section{a. Using Computers for Lectures}

We started using computers for lectures at an early stage: more than 10 years ago we used a "Sinclair Spectrum" with output on two TV monitors. Besides the list of main. ideas of each course we also used a few simple programs illustrative for understanding some basic notions such as: sampling a biosignal, what does a frequential analysis do, what is a spectrum, the principle of computer tomography, the role of the grid in scintigraphy, etc. [8].

Nowadays we use a notebook connected to a LCD panel; almost all lectures are presented this way. We have inserted demo sequences from other programs. Our LCD has alșo a video input and our students can appreciate the video film made by DTI [9] about using computers in healthcare.

Our experience shows that students are sensitive to the variety of methods used for teaching and we should not underestimate the impact a specific teaching method will have on the students. b. Designing Specific Computer Programs for Laboratory Works

Our original intention was that this paper would be dedicated only to this feature. This topic developed into one of our major objectives because the existing programs are not satisfactory. For one who carefully designs the objectives of his courses, it becomes clear that a few commercially available programs will fulfill educational requirements. The demos which accompany this software are sometimes useful but generally they are not interactive. We have a very clear objective: to offer students an equivalent laboratory program for any topic presented in lectures. The computer programs designed for our laboratory classes referred to:

- biosignal acquisition and processing (similar to that presented in [10]),

- biostatistics: generating data with known distribution and parameters [11], which can also be used for analysis with various statistical packages,

- simulation of biological processes [12-14]; we noticed the quality of understanding the phenomena after "playing" with various parameters and seeing their influence on the effects.

We are now preparing programs for laboratory work on information systems (in primary care) and pattern recognition.

\section{Medical Informatics for Dentistry School}

To date, dental informatics focused on a limited range of applications, mainly concerning administrative tasks: computer-based dental patient record and clinical databases (without decision-support systems). Very few of the specialists in dentistry followed formal training in medical informatics and, even when they did, dental informatics was briefly represented in the curriculum. Most of them taught themselves to use a computer and, unfortunately, in Rumania very few specialists have had the opportunity to collaborate in application development - we mean applications to be used beyond the walls of their own institution and not restricted to the current necessities or limited by the available local resources.

We took over the medical informatics curriculum for the students in dentistry, but tried toemphasize the specific features: signal and image processing for dental aspects, the use of CAD/ CAM techniques in prosthetics, quality assessment of medical activities, decision-supportfordentistry, visualizations and simulations specific for facial surgery. As already mentioned, because both medical knowledgeand informatics change quickly we have to provide students with a good grounding in problem solving and information retrieval skills, rather than give them "aniversal" solutions or knowledge [15]. They should see informatics and computers as useful tools to improve access to data and knowledge bases, communication with their colleagues and patients, and to improve the quality of medical practice.

Even if our teaching strategy is still "teacher-centered", we tried to mix it with laboratory problem-oriented work: biostatistics, computer-based dental patient record, databases for the dentist's office.

\section{Informatics for Pharmacy School}

Our pharmacy students have their course on medical informatics in the first year of study (second term) after a course on mathematics and biostatistics(first term). The informatics course has 15 hours of lectures and 15 hours of laboratory work, which are insufficient to cover the topics normally presented in similar courses in other uni- 
versities. We found few papers [16] dealing with medical/pharmacy informatics education for students of pharmacy schools.

Students should understand the pasition and role of a pharmacist in a health information system, become familiar with concepts of health information, appreciate the capabilities of a pharmacy information system (drug ordering, stock control, drug interaction checking, billing, drug delivery, therapeutic monitoring) and understand the use of computers for drug design and pharmacological studies (pharmacokinetics, pharmacodynamics, toxicology).

In order to reach these objectives we designed a curriculum introducing two specific lectures: one on pharmacokinetics (principles, formal description of multicompartmental systems, computer programs [17]), pharmacodynamics (modelling drug receptor interaction, dose-response curves processing [18]), principles of drug design and QSAR (Quantitative Structure Activity Relation) studies [19]; and the otherdedicated to drug interaction studies [20] and pharmaceutical information systems [21].

For laboratory work we use a specific program for pharmacokinetics [22] and our programs for pharmacodynamics [18], comprising a simulation of drug-receptor interaction and processing of dose-response curves. For QSAR studies we use a demo version for 3D molecular structures.

We estimate that we have insufficient software for specific pharmacological topics. Also, there is still a low level of computer implementation in pharmacy units. However, it is expected that this sector, which is now largely privatized, will developquickly.

\section{Graduation Theses}

In Rumania, students have to preare a graduation thesis as a problem- based project. Medical students carry out the project during the last year of study and can choose the coordinating professor and department according to their preferences. Each year, we have 2-6 graduating students who choose to work co-ordinated by our staff. Most of them graduated from high schools with informatics included in the mandatory curricula.

We tried to define project themes related to fields we are doing research on, but we also allow the students to choose subjects which fit their particular interests and can help them in their future careers. Furthermore, we find this a good opportunity to develop a stronger collaboration between technical schools and medical schools, and to encourage our students to have a multidisciplinary approach to medical informatics and to develop skills suitable for "life-long learners". We collaborated mainly with the clinical departments of our university and with the Polytechnic University of Timisoara, especially with the School of Computers and Control Engineering. We also had "mixed" teams, with medical and technical graduating students, trying to bridge the gap between healthcare specialists and informaticians or computer engineers, and to compensate for the lack of medical informatics specialization in our universities.

Ourstudents collaborated especially for building medical databases for some clinics (e.g. clinics of Hematology) and biostatistical processing of data, but also in other areas of research: gait analysis (Mental Health Laboratory of Satu-Mare and Timisoara School of Computers), computer-aided learning application on neuro-ophthalmology for students and young physicians (clinics of Neurology and Ophthalmology), simulations of pharmacological processes (School of Pharmacy), 3Dvisualization of face and jaw and study of malocclusions (School of Dentistry and School of Computers), simulation of visual processes - stereovision (School of Computers), a little knowledge-database (School of Dentistry).

The students have to present their work and defend the thesis at an oral examination. Furthermore, we encouraged our graduating students to present their results at the annual Rumanian National Conferences on Medical Informatics.

\section{Medical Informatics for Postgraduate Studies}

While undergraduate and Ph.D. studies are organized by the Ministry of Education, other postgraduate medical studies are organized by the Ministry of Health. Within their programs we have two modules per year, each of them having 15 hours of lectures and 30 hours of laboratory work over a period of six weeks, with groups of 712 students. These courses are optional; they are attended especially by physicians who had no such classes during their studies. We mainly followed the structure and contents of the courses and laboratory works of medical students (but it does not comprise the chapter on biostatistics). We estimate that the real requirement for such courses will soon increase since the investment plans for the introduction of a large number of computers in several medical units have already started, and it will probably become an educational program on anational scale.

\section{Doctoral Level}

The doctoral level in medical informatics in Rumania started in 1992 with four students and up to now there are 10 students with different backgrounds: 2 physicians, 4 mathematicians and 4 engineers. There are several unsolved problems within this respect.

We should first mention that ac- 
cording to the Education Law of Rumania, the doctoral level, as a postgraduate form of education, is organized by universities and the coordinators are appointed nominally by the National Council for Attesting Titles, Diplomas and University Certificates (CNATDCU). There are only about 12 major fields in which one can get a $\mathrm{Ph}$.D. in Rumania, including mathematics, physics, engineering, medicine etc. Each major field is divided into specialities, with a list of approved specialities for each field. But only the name of the field, not the speciality, is written on the Ph.D. diploma. A postgraduate student working for a Ph.D. is registered at the university where his coordinator works, in our case at the University of Medicine and Pharmacy in Timisoara; however, a medical university may only award "Ph.D. in medicine" and not in e.g. mathematics or in engineering. Thus, only medical doctors can defend their theses at a medical university. For our Ph.D. students in medical informatics who are not MDs a provisory solution was proposed: the dossier of the candidate would be transferred to an appropriate university accredited to award the Ph.D. title in the candidate's field. Second, we should also mention that most of the Ph.D. students keep their full-time jobs and their preparation is done during other everyday tasks - it is even called "doctoral stage without attending program". The doctoral stage starts with an admittance colloquium and lasts about four years comprising two phases: examinations and reviews strictly restricted to two years, and thesis preparation taking another two years.

The coordinator has to decide on the number and the topics of examinations and reviews as well as establishing the contents and the references to be used for preparation of the exams. The examination is performed by a commission of at least four invited professors, specialists in the topic or speciality of the exam. The reviews are presented and discussed at the meetings of the department and refereed by a commission similar to that for the examinations. There are no restrictions or rules concerning the topics subjected to become the name of an exam, but this usually follows the traditions and exigencies associated with Ph.D. preparation. The thesis has to be reviewed by at least five professors from different universities, specialists in the speciality or topic of the thesis. The candidate is also required to have at least two published papers on the topic of the thesis. All commissions, for examinations, reviews and thesis are proposed by the coordinator and have to be approved by both the Professorial Council of the school and by the Senate of the university. The title is validated by CNATDCU.

A recent meeting at the ministerial level proposed to improve the doctoral systemand, hopefully, will solve some of these problems.

\section{Future Perspectives}

Our efforts are oriented towards a stronger integration of our discipline within the global framework of medical education; better communication with colleagues from various disciplines within our university; and stronger links with similar departments, both domestic and international. As a priority we intend to emphasize scientific research activities.

We expect that our new LAN and the multimedia laboratory will markedly improve the infrastructure facilities for educational purposes.

Our links with similar departments at other medical universities are good; we periodically meet during national medical informatics conferences; we try to keep similar curricula, exchange programs, and share experience. We also try to attend international events, as far as financial resources allow. We participated in EuroMISE courses organized in Prague [6] and participated in a TEMPUS project. It is hoped to extend our contacts; perhaps via Euro. pean programs and bilateral agreements.

\section{References}

1. Van Bemmel JH, Hasman A, SolletPCGM, Veth AFL. Training in medical informatica Comput Biomed Res 1983;16;414-32.

2. Salamon R, Protti D, Moehr J, eds. Inten national Symposium in Medical Informatic and Education. Victoria BC: Universit Press, 1989: 505-30.

3. Ingram D, Murphy J. Integrating informatics into the undergraduate currick lum: A report on a pilot project. In: Van Bemmel JH, McCray AT, eds. Yearbook of Medical Informatics. Stuttgart: Schattauer Verlag, 1996:116-21.

4. EDUCTRA members. Education and training in health informatics: Guidelines for European curricula. In: Hasman A, Albert A, Wainwright P, Klar R, Sosa $\mathbf{M}$, eds. Education and Training in Health Informatics in Europe. Amsterdam: IOS Press, 1995: 27-50.

5. Van Bemmel JH. Data, information and knowledge for education in medical informatics. In: Salamon R, Protti D, Moehr J. eds. International Symposium on Medi. cal Informatics and Education. Victoria BC: University Press, 1989: 495-9.

6. Zvarova J. Medical informatics education in the EuroMISECourses. In: Greenes RA, Peterson HE, Protti DJ, eds. Proceedings of the $8^{\text {th }}$ World Congress of Medical Informatics. MEDINFO'95. Amsterdam North-Hollland, 1995:1162-4.

7. Piemme TE, Pincetl PS, Malakoff GL, Clyman SG, Julian ER, Case SM, Swanson DB, Cotton KE, El Bayoumi J, Chang L. Use of Expert Judgment to Validate a Scoring Algorithm in Assessing Performance on Computer Simulations. In: Lun KC, Degoulet P, Piemme TE, Rienhoff O (eds). MEDINFO'92. Amsterdam: North-Holland; 1992: 1128-33.

8. Mihalas GI. TIM - A set of programs for teaching medical informatics. In: Van Bemmel JH, Zvarova J (eds). Knowledge. Information and Medical Education. Amsterdam: North-Holland; 1991: 95-100.

9. Jones B, Shipton J. Images of Healthcare Technology (video). Department of Trade and Industry. London: QUAI Interactive. 1996.

10. Cammann H, Michel J. Aspects of acquirsition and processing of measurable biomedical variables in teaching Medicat 
Informatics. In: Van Bemmel JH, Zvarova $\mathrm{J}$, eds. Knowledge, Information and MedicalEducation. Amsterdam: North-Holland, 1991:101-7.

11. Mihalas GI, Lungeanu D. Computer simulation programs for teaching biostatistics to medical students. In: Richards B, ed. Current Perspectives in Healthcare Computing. Surrey: BJHC Ltd., 1996:353-8.

12. Mihalas GI, Lungeanu D, Kigyosi A, Vernic C. Classification criteria for simulation programs used in medical education. In: Greenes RA, Peterson HE, Protti DJ, eds. Proceedings of the $8^{\text {th }}$ World Congress on Medical Informatics. MEDINFO'95. Amsterdam: North-Holland, 1995:120913.

13. Mihalas GI. Computer simulation of membrane phenomena. In: Salamon R, Blum B, Jorgensen M, eds). Proceedings of the $5^{\text {th }}$ World Congress on Medical Informatics. MEDINFO'86. Amsterdam: North Holland, 1986:1118.

14. Mihalas GI. Criteria for computer simulation of drug-receptorinteraction. In: Fedina L, Kanyar B, Kocsis B, Kollai M (eds). Mathematical and ComputationalMethods in Physiology.Adv. Physiol. Sci. Vol. 34 New York: Pergamon Press, 1981:87-91.
15. Abbey LM, Zimmerman JL, eds. Dental Informatics: Integrating Technology into the Dental Environment. New York: SpringerVerlag, 1992.

16. Radojka ZH. The Role of Medical Informatics in Pharmacy: A Canadian Perspective. In: Salamon R, Protti D, Moehr J, eds. International Symposium on Medical Informatics and Education. Victoria BC: University Press, 1989:194-5.

17. Jelliffe RW, Bayard D, Shumitzky A, Milman M, Van Guilder M. Pharmacoinformatics: more precise drug therapy. In: Greenes RA, Peterson HE, Protti DJ, eds. Proceedings of the $8^{\text {th }}$ World Congress on Medical Informatics. MEDINFO'95. Amsterdam: North-Holland, 1995:110610.

18. Mihalas GI, Simon Z. Computers in studies of drug-receptor interaction. In: Van Bemmel JH, Gremy F, Zvarova J, eds. Medical Decision Making. Amsterdam: North Holland, 1985:325-30.

19. Simon Z, Chiriac A, Holban S, Ciubotariu D, Mihalas GL Minimum Steric Difference. The MTD Method for QSAR Studies. Letchworth, England: Research Studies Press, 1985:3-79.

20. Solomon WD, Heatfield HA. Conceptual modeling used to represent drug interactions. In: Barahona P, Veloso M, Bryant J, eds. Proceedings MIE'94. Lisbon, 1994: 186-90.

21. Sanz F, Loza MI, Ahlgrimm ED, Baetens $P$, Cuypers J, Cranz H, Coronel $M$, Fernandez-Lopez JL, De Mora A, SosaIudicissa M. Telematics in community pharmacies to support responsible selfmedication. The TESEMED project. In: Brender J, Christensen JP, Scherrer JR, McNair P (eds). Proceedings MIE'96. Amsterdam: IOS Press, 1996:141-5.

22. Heinzel G, Woloszczak R, Thomann P. Pharmacokinetic and Pharmacodynamic Data Analysis System for the PC (TOPFIT 2.0). Stuttgart: Gustav Fischer; 1993: 2.5$60,3.6-133$.

Address of the authors:

George I Mihalas, Diana Lungeanu, University of Medicine and Pharmacy, Department of Medical Informatics, P-ta Eftimie Murgu no. 2, 1900 Timisoara,

Rumania.

e-mail:mihalas@asmt.sorostm.ro dlungeanu@umft-dim.sorostm.ro 